\title{
Silver coordination polymers based on newly designed bis- (cyanobenzyl)bipipyridine ligand: synthesis, anion exchange, guest inclusion, electrochemical and photoluminescence properties
}

Sandipan Roy, Hatem M. Titi, Bharat Kumar Tripuramallu, Nisith Bhunia, Roli Verma and Israel Goldberg*

School of Chemistry, Sackler Faculty of Exact Sciences, Tel-Aviv University, Ramat-Aviv, 69978 Tel-Aviv, Israel. E-mail: sandipanroy06@gmail.com, goldberg@post.tau.ac.il

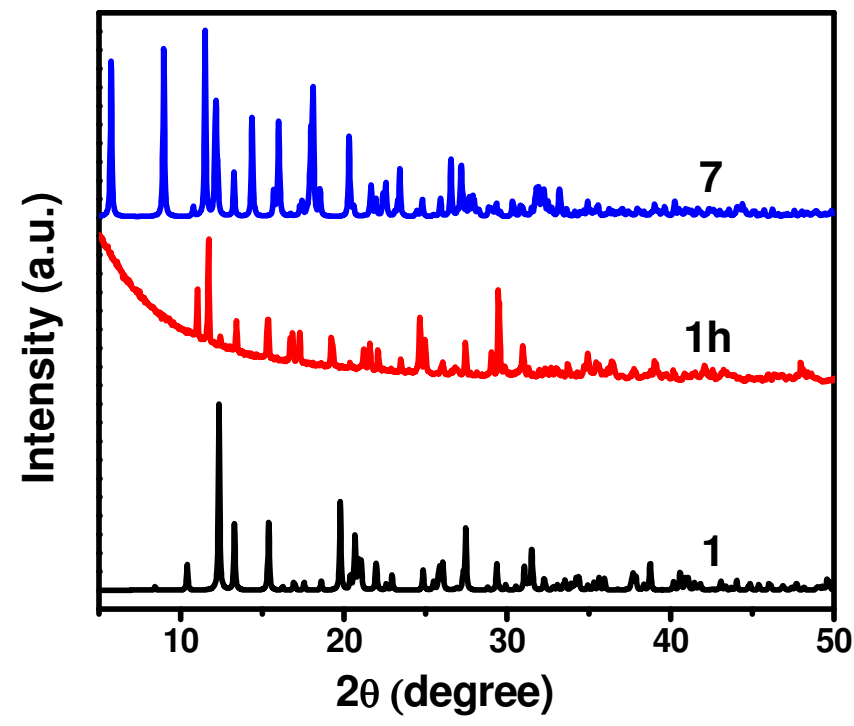

Figure S1. PXRD patterns of 7 after anion-exchange employing $3 \mathrm{M} \mathrm{NaNO}_{3}$ aqueous solutions for 1h. The data shown in the top and bottom represent the PXRD patterns for $\mathbf{7}$ and $\mathbf{1}$ as prepared by direct synthesis. 

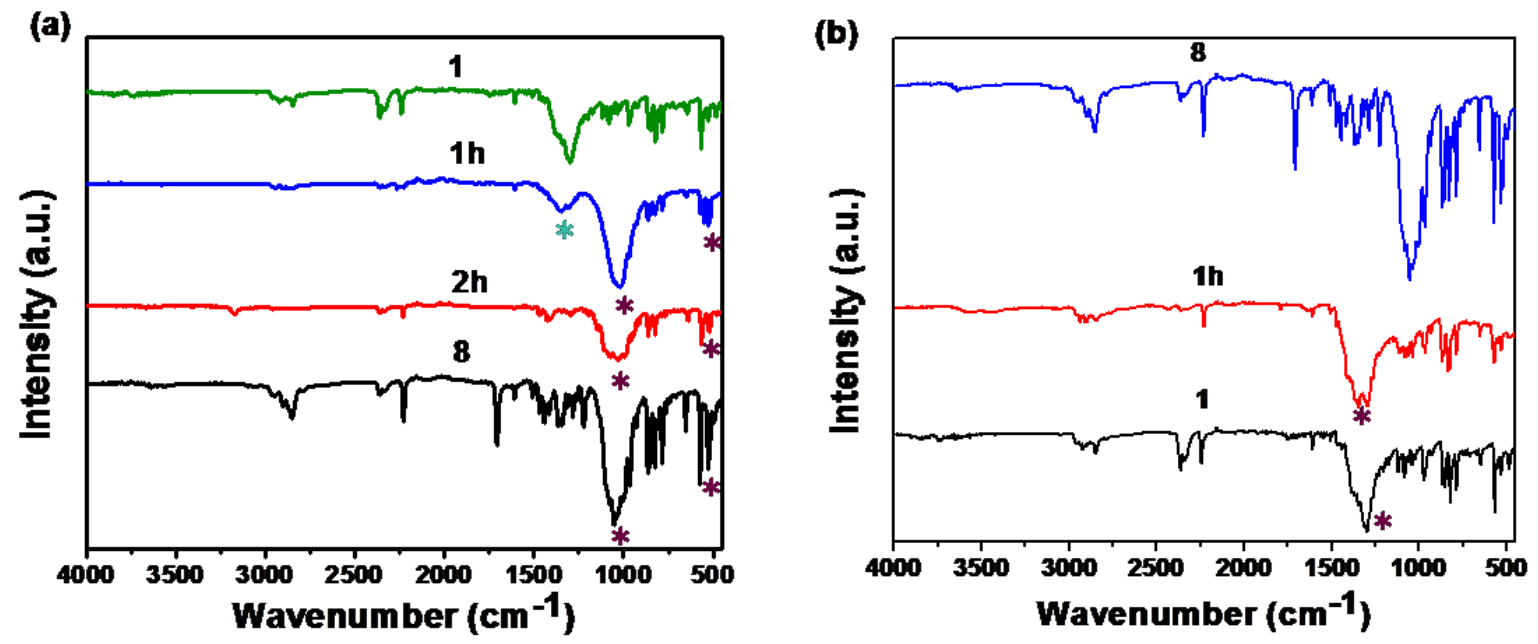

Figure S2. (a) IR spectra of 1 after anion-exchange employing $3 \mathrm{M} \mathrm{NaBF}_{4}$ aqueous solution for 1 and $2 \mathrm{~h}$. The top and bottom curves represent the IR spectra for $\mathbf{1}$ and $\mathbf{8}$ as prepared by direct synthesis. (b) IR spectra of 8 after anion-exchange employing $3 \mathrm{M} \mathrm{NaNO}_{3}$ aqueous solution for 1 h. The top and bottom curves represent the IR spectra for $\mathbf{8}$ and $\mathbf{1}$ as prepared by direct synthesis.

(a)

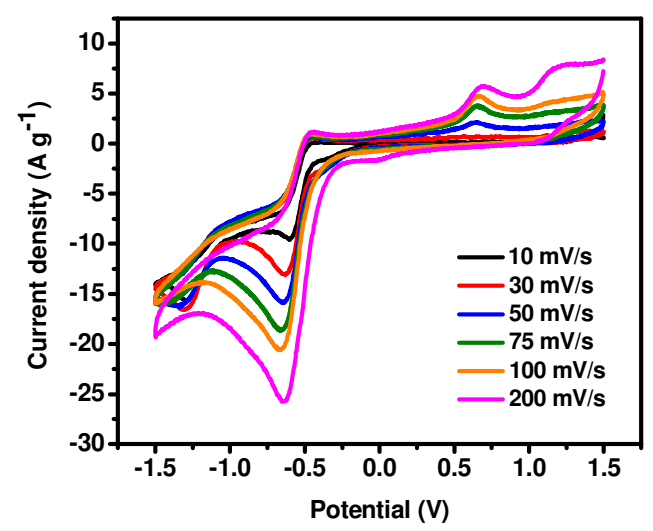

(b)

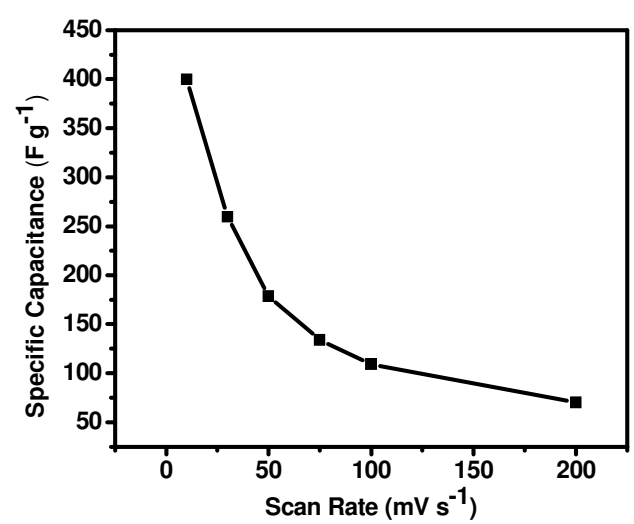

Figure S3. (a) Cyclic voltammograms of ligand $\mathbf{L}$ at different scan rates in $\mathrm{CH}_{3} \mathrm{CN}$. (b) Variation of specific capacitance of $\mathbf{L}$ with scan rate. 
(a)

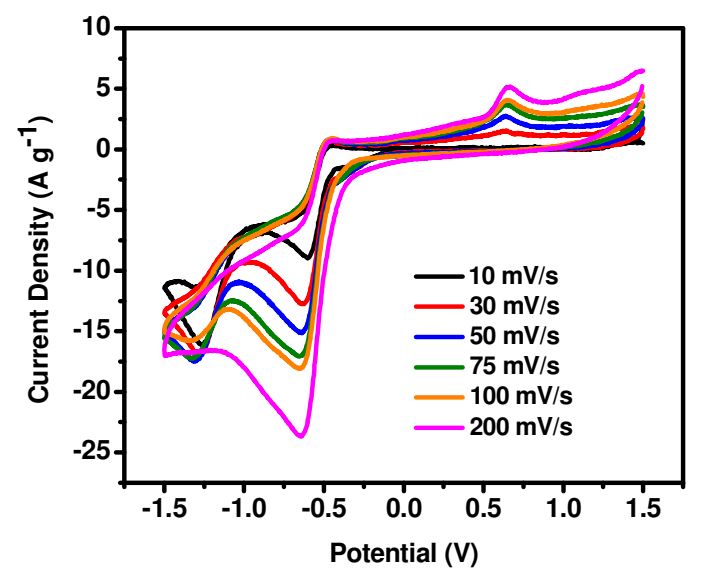

(b)

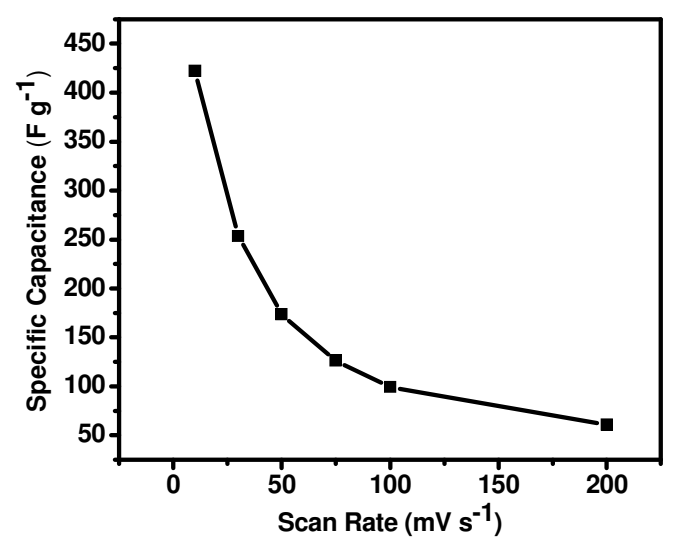

Figure S4. (a) Cyclic voltammograms of complex 1 at different scan rates in $\mathrm{CH}_{3} \mathrm{CN}$. (b) Variation of specific capacitance of $\mathbf{1}$ with scan rate.

(a)

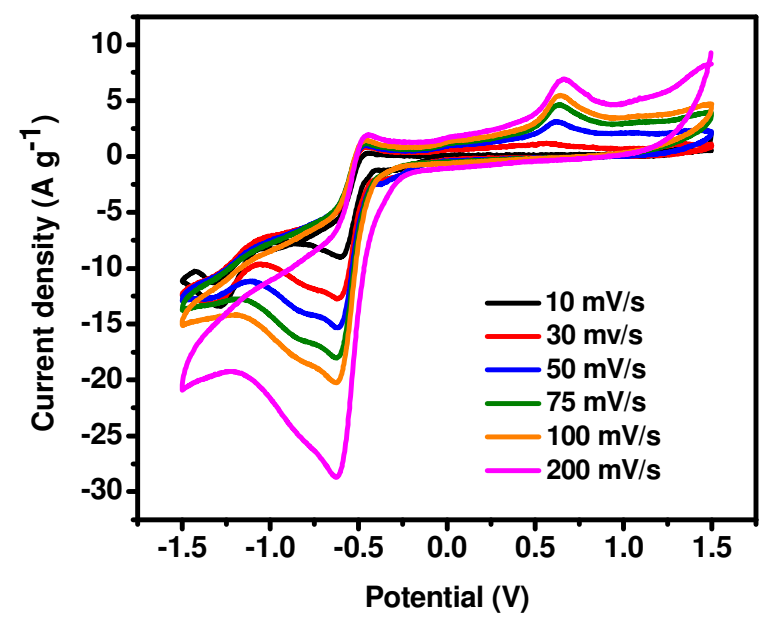

(b)

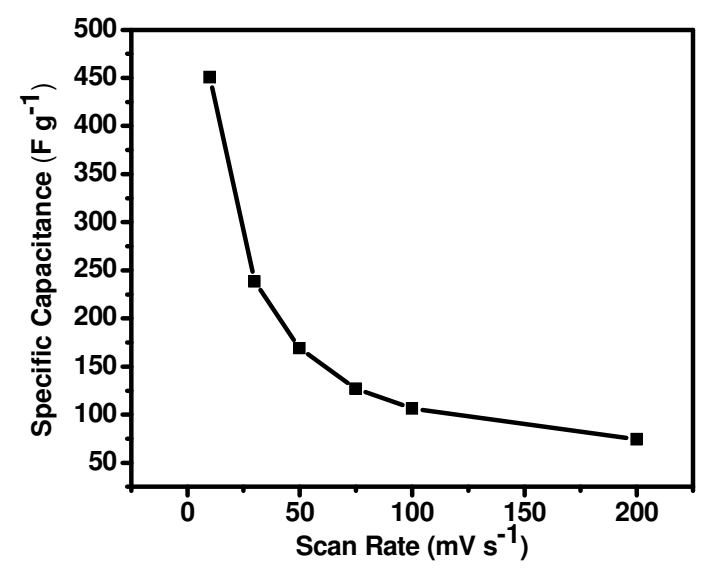

Figure S5. (a) Cyclic voltammograms of complex $\mathbf{3}$ at different scan rates in $\mathrm{CH}_{3} \mathrm{CN}$. (b) Variation of specific capacitance of $\mathbf{3}$ with scan rate. 
(a)

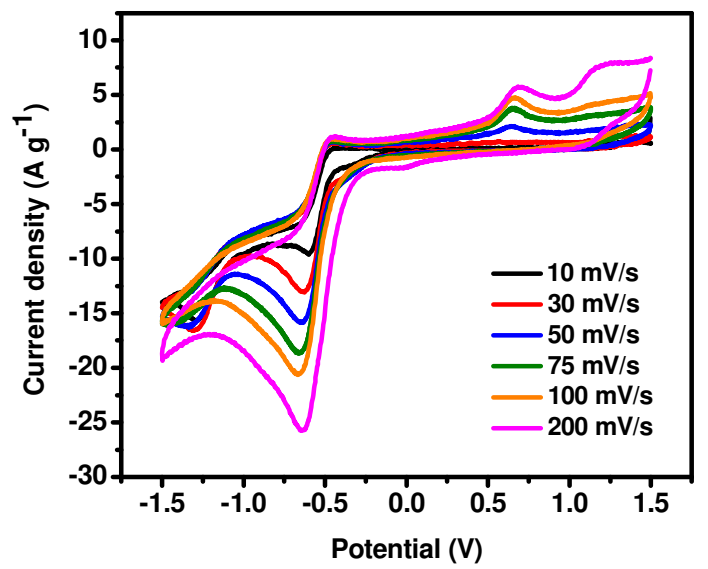

(b)

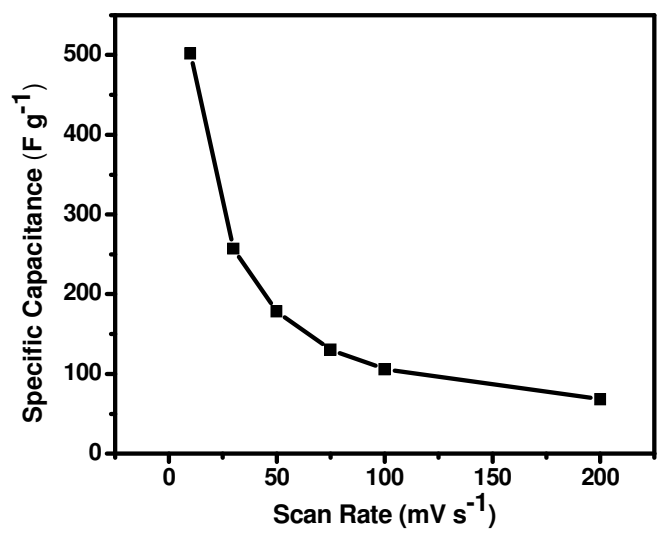

Figure S6. (a) Cyclic voltammograms of complex 4 at different scan rates in $\mathrm{CH}_{3} \mathrm{CN}$. (b) Variation of specific capacitance of $\mathbf{4}$ with scan rate.

(a)

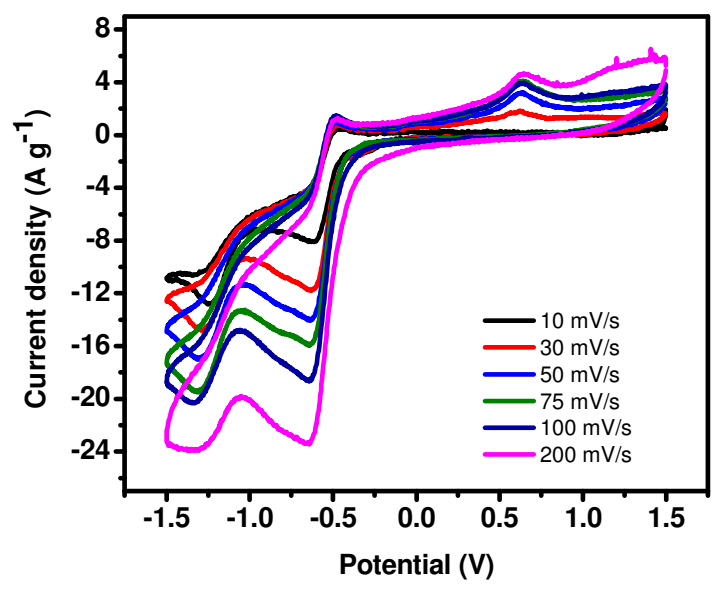

(b)

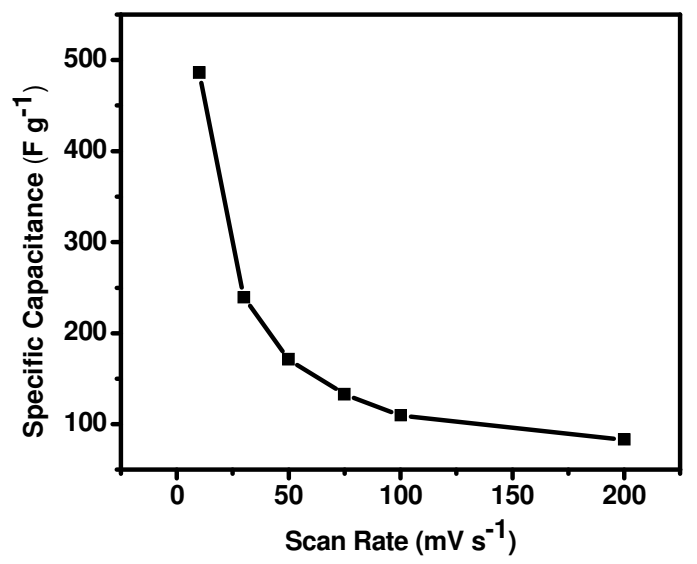

Figure S7. (a) Cyclic voltammograms of complex 7 at different scan rates in $\mathrm{CH}_{3} \mathrm{CN}$. (b) Variation of specific capacitance of $\mathbf{7}$ with scan rate. 
(a)

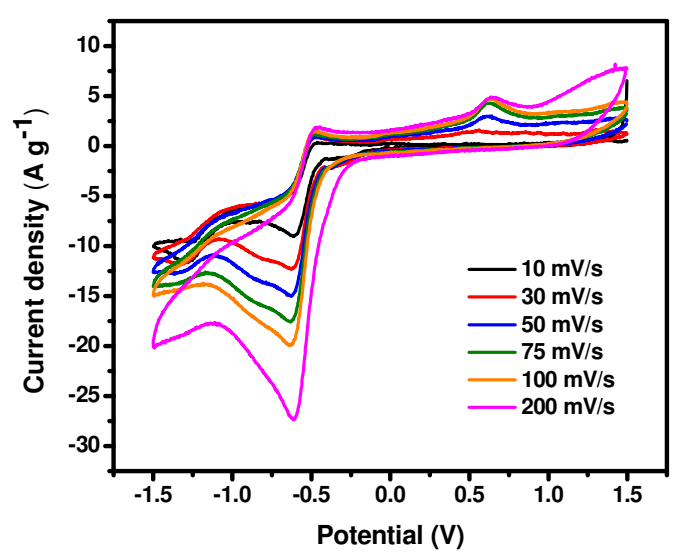

(b)

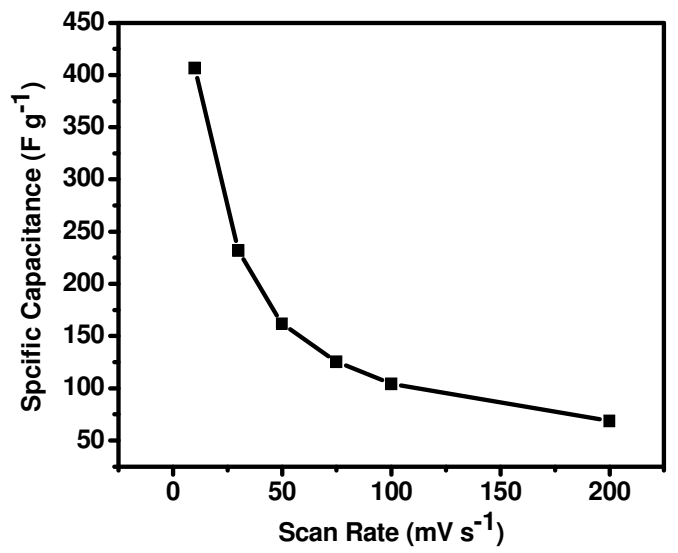

Figure S8. (a) Cyclic voltammograms of complex 8 at different scan rates in $\mathrm{CH}_{3} \mathrm{CN}$. (b) Variation of specific capacitance of $\mathbf{8}$ with scan rate.

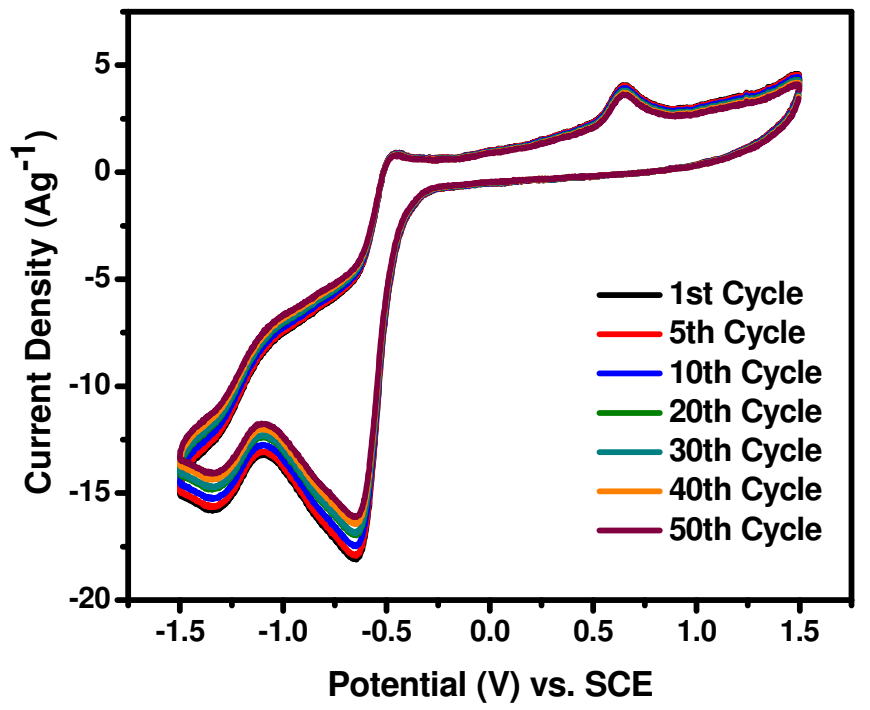

Figure S9. Cyclic voltammograms of complex 1 up to 50 cycles (in $\mathrm{CH}_{3} \mathrm{CN}$ ), using $0.2 \mathrm{M}$ EMIM TFSI as the supporting electrolyte. 